\title{
Diseño Y modelado del robot PA-10 virtual para aplicaciones quirúrgicas
}

\author{
Design and modeling of a virtual PA-10 robot for surgical \\ applications
}

Desenho e modelamento do robô PA-10 virtual para aplicações cirúrgicas

Fecha de recepción: 07 de febrero de 2016

Fecha de aprobación: 18 de abril de 2016
Carlos Eduardo Fernández-Riomalo* Héctor Andrés Guástar-Morillo* Oscar Andrés Vivas-Albán ${ }^{* *+}$

\section{Resumen}

El trabajo presenta la implementación de un simulador quirúrgico virtual para operaciones de laparoscopia utilizando asistentes robóticos. Para este tipo de simuladores son necesarios tres robots: un robot portaendoscopio (en este caso se utiliza un robot Hibou) y dos robots quirúrgicos (en este caso un robot Lapbot y un robot tipo PA10). Los tres robots son manejados por joystick en un ejercicio de extracción de vesícula biliar (colecistectomía), y se incluyen en el ejercicio algoritmos de deformación que modifican los órganos al contacto del órgano terminal del robot PA-10, imprimiéndole mayor realismo al simulador. Los resultados proporcionan la base de un simulador quirúrgico utilizando tres robots asistenciales, en un ejercicio común en laparoscopia, ideal para el entrenamiento de nuevos cirujanos.

Palabras clave: laparoscopia, modelado de robots, robot virtual, robótica quirúrgica, simulador quirúrgico.

\section{Abstract}

This paper shows the implementation of a PA-10 Mitsubishi robot on a surgical virtual simulator for laparoscopic surgeries by using surgical assistant robots. For the proposed simulator three robots are necessary: An endoscopeholder robot (in this case the Hibou robot), and two surgical robots (in this case a Lapbot robot and the designed PA-10 robot). The three robots are manipulated by a joystick device on a gallbladder ablation exercise

\footnotetext{
* Universidad del Cauca (Cauca - Popayán, Colombia). carlosfr@unicauca.edu.co.

** Universidad del Cauca (Cauca - Popayán, Colombia). hectorgm@unicauca.edu.co.

*** Ph.D. Universidad del Cauca (Cauca - Popayán, Colombia). avivas@unicauca.edu.co.
} 
(cholecystectomy). Deformation algorithms are included to provide greater realism, when the end-effector of the robot collides with the PA-10 virtual robot, abdominal organ. Results provide the base of a surgical virtual simulator using three assistant robots on a common laparoscopic surgical exercise, that can be used for training new surgeons.

Keywords: maparoscopic, modeling robot, surgical robotics, surgical simulator, virtual robot.

\section{Resumo}

O artigo apresenta a implementação de um simulador cirúrgico virtual para operações de laparoscopia utilizando assistentes robóticos. Para este tipo de simuladores são necessários três robôs: um robô porta endoscópio (neste caso se utiliza um robô Hibou) e dois robôs cirúrgicos (neste caso um robô Lapbot e um robô tipo PA-10). Os três robôs são controlados por joystick em um exercício de extração de vesícula biliar (colecistectomia), e se incluem no exercício algoritmos de deformação que modificam os órgãos ao contato do órgão terminal do robô PA-10, imprimindo-lhe maior realismo ao simulador. Os resultados proporcionam a base de um simulador cirúrgico utilizando três robôs assistenciais, em um exercício comum em laparoscopia, ideal para o treinamento de novos cirurgiões.

Palavras chave: laparoscopia, modelamento de robôs, robô virtual, robótica cirúrgica, simulador cirúrgico.

Cómo citar este artículo:

[1] C. E. Fernández-Riomalo, H. A. Guástar-Morillo and O. A. Vivas-Albán, "Diseño y modelado del robot PA-10 virtual para aplicaciones quirúrgicas", Fac. Ing., vol. 25 (42), pp. 21-32, may.-ago. 2016. 


\section{INTRODUCCIÓN}

Con el paso de los años, el uso de la robótica en el campo de la medicina se ha incrementado considerablemente. La precisión y exactitud que otorga el uso de robots asistenciales brinda grandes ventajas en cirugía cardíaca, gastrointestinal, pediátrica, neurocirugía y, específicamente, en cirugía mínimamente invasiva. Los retos que este último tipo de cirugía impone al cirujano (fatiga, temblor en las manos, efecto de inversión mano-ojo, pérdida de sensación táctil) hacen deseable la ayuda de una máquina.

Dentro de la cirugía mínimamente invasiva se destaca la laparoscopia [1-2], procedimiento relativamente reciente practicado a través de pequeños orificios en la zona abdominal, por donde el cirujano introduce los instrumentos quirúrgicos y una cámara de video (endoscopio). Este procedimiento, si bien trajo grandes ventajas para el paciente (menor probabilidad de infecciones, menor dolor, menor tiempo en el hospital), planteó mayores retos para el cirujano, pues no es lo mismo realizar una operación con el abdomen abierto que a través de pequeños orificios.

Dentro de los robots asistentes en cirugía laparoscópica sobresale el Da Vinci [3-4], que tiene tres brazos quirúrgicos manejados a distancia por el cirujano, $\mathrm{y}$ un brazo portaendoscopio, el cual envía una imagen del interior del abdomen del paciente. Otros asistentes quirúrgicos que solo manejan el endoscopio son el robot Lapman [5], el robot Ensoassist [6] y el robot Aesop [7].

Como se anotó anteriormente, los retos para el cirujano son cada vez mayores; de ahí la importancia del entrenamiento en estas nuevas tecnologías; los simuladores por computador ofrecen sistemas con elevado realismo, facilitando el entrenamiento para la práctica de la laparoscopia. Existen varios tipos de entrenadores; los de tipo físico [8-10] tienen un torso artificial real e instrumentos reales; los de tipo virtual (como el LapMentor [11], el Mentice [12], el LapSim [13] y el Da Vinci Skills Simulator [14]) se basan, sobre todo, en simulación en computador, con importantes características de realismo como el sangrado al cortar un tejido o el humo producido por el cauterizador.

La Universidad del Cauca, en Popayán, Colombia, ha venido trabajando desde hace unos años en una versión de simulador quirúrgico virtual [15] que tiene dos robots: uno quirúrgico (llamado Lapbot [16]) y otro portaendoscopio (llamado Hibou [17]). El simulador ha sido programado para realizar una operación de colecistectomía (extracción de la vesícula), utilizando para ello un dispositivo joysctick, que maneja tanto la cámara virtual (robot Hibou), con el fin de ubicarla sobre el lugar a intervenir, como el robot que porta los instrumentos quirúrgicos necesarios (robot Lapbot con los instrumentos: bisturí, pinza y cauterizador).

Sin embargo, para completar el simulador se necesita un segundo robot portainstrumentos. Como los dos robots ya cuentan con un prototipo real construido hace un par de años, se necesita otro robot que pueda trabajar junto con el Lapbot actual. La opción más fácil era duplicar dicho robot, pero con el fin de probar y comparar su desempeño con otro tipo de robot se decidió implementar un robot tipo comercial dentro del simulador actual, para en un futuro cercano construir así mismo ese prototipo. Se escogió, por lo tanto, el robot PA10 de Mitsubishi [18-19], utilizado tanto en el medio industrial como en la investigación médica.

Este trabajo muestra, entonces, el simulador quirúrgico completo, incluyendo algoritmos de contacto y deformación, lo cual le agrega mayor realismo. El aporte principal del artículo es completar un simulador quirúrgico robotizado, primero de su tipo en Colombia, el cual permitirá no solo el entrenamiento de los nuevos cirujanos, sino introducir en el país los nuevos avances en robótica quirúrgica que se están desarrollando en el mundo.

El artículo está dividido de la siguiente manera: la segunda sección presenta la descripción de los robots Lapbot e Hibou; la tercera, el diseño y modelado del robot PA-10; la cuarta, el software desarrollado; la quinta, los resultados obtenidos, y la sección final, las conclusiones más importantes de este trabajo.

\section{Robots Lapbot e Hibou}

El robot Lapbot [16] tiene nueve grados de libertad, con seis articulaciones activas y tres pasivas, lo que le asegura el mantenimiento de un punto fijo al pasar por el trocar (orificio de la cavidad abdominal). Todas las articulaciones son de tipo rotacional, a excepción de la primera, que es prismática. La Fig. 1 muestra la estructura cinemática del robot Lapbot, donde el anillo entre las articulaciones 7 y 8 representa el paso por 
la cavidad abdominal. $D_{i}$ y $R_{i}$ hacen referencia a las distancias según la representación de Dombre y Khalil [20].

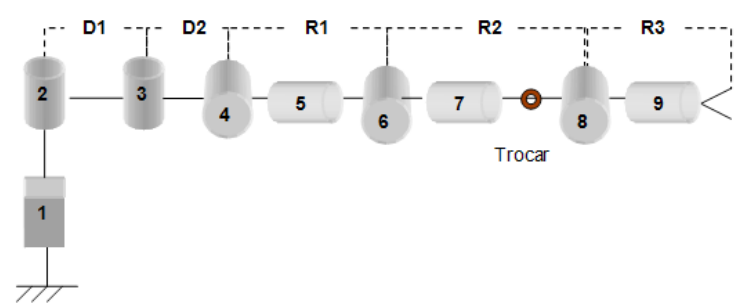

Fig. 1. Arquitectura robot Lapbot.

El robot Hibou [17], por su parte, está diseñado para soportar la cámara o endoscopio en el interior del abdomen del paciente; está compuesto por siete articulaciones rotacionales: cinco activas y dos pasivas, lo que asegura, igualmente, el mantenimiento de un punto fijo sobre la cavidad abdominal. La Fig. 2 muestra la estructura de este robot, ubicándose el trocar entre las articulaciones 5 y 6 .

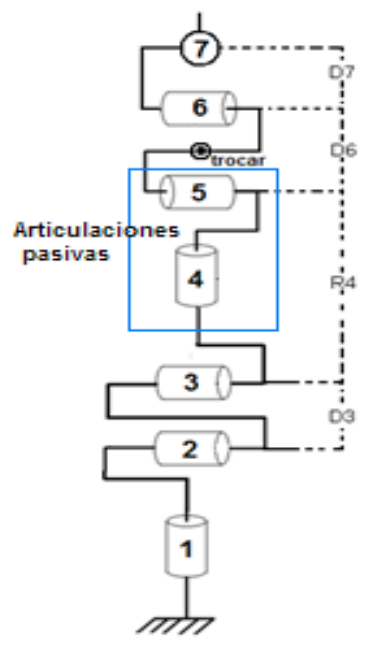

Fig. 2. Arquitectura robot Hibou.

Como se comentó anteriormente, estos dos robots cuentan ya con su primer prototipo real (Fig. 3).

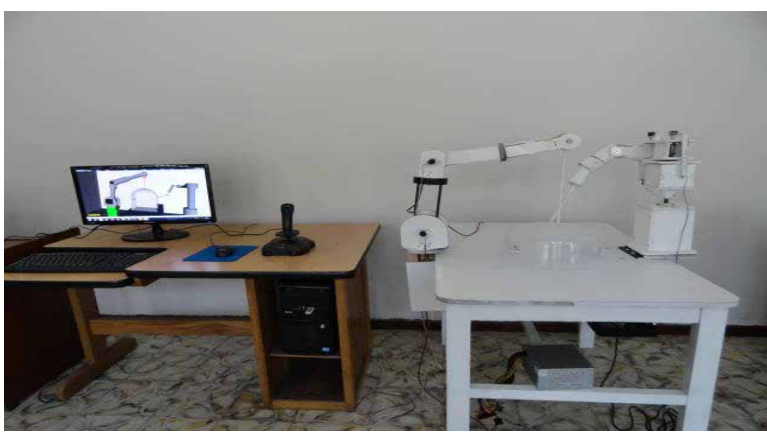

Fig. 3. Prototipo de los robots Lapbot e Hibou.

\section{Diseño Y MOdelado del ROBOT PA-10}

\section{A. Parámetros del robot PA-10}

El PA10 es un robot de siete grados de libertad, con siete articulaciones rotoides y una muñeca de ejes concurrentes, fabricado por Mitsubishi y muy popular en la automatización de procesos (Fig. 4).

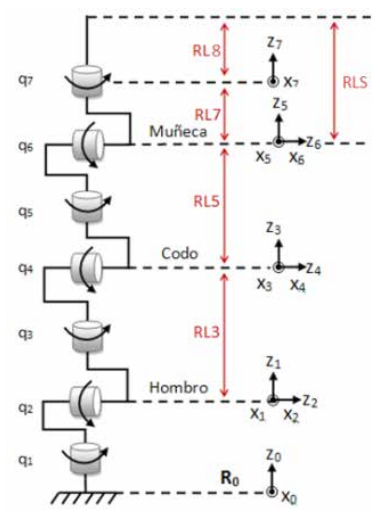

Fig. 4. Estructura cinemática del robot PA-10.

Según el análisis de Dombre y Khalil [20], se obtiene la tabla de parámetros geométricos respectiva (Tabla $1)$, donde $j$ designa el número de la articulación; $\sigma_{j}$ se refiere al tipo de articulación ( 0 para las articulaciones rotacionales, 1 para las prismáticas); $\alpha$ se refiere al ángulo entre los ejes $z ; d_{j}$ representa la distancia entre los ejes $z ; \theta_{j}$ simboliza la variable articular rotacional o un ángulo entre los ejes $x_{i}$, y, por último, $r_{j}$ simboliza la variable articular prismática o una distancia entre los ejes $x_{i}$. 


\section{TABLA 1}

PARÁmetros GEOMÉTRICOS DEL ROBOT PA-10

\begin{tabular}{c|c|c|c|c|c}
\hline$j$ & $\sigma_{j}$ & $\alpha_{j}$ & $d_{j}$ & $\theta_{j}$ & $r_{j}$ \\
\hline 1 & 0 & 0 & 0 & $\theta_{1}$ & 0 \\
\hline 2 & 0 & -90 & 0 & $\theta_{2}$ & 0 \\
\hline 3 & 0 & 90 & 0 & $\theta_{3}$ & RL3 \\
\hline 4 & 0 & -90 & 0 & $\theta_{4}$ & 0 \\
\hline 5 & 0 & 90 & 0 & $\theta_{5}$ & RL5 \\
\hline 6 & 0 & -90 & 0 & $\theta_{6}$ & 0 \\
\hline 7 & 0 & 90 & 0 & $\theta_{7}$ & RL7 \\
\hline 8 & 0 & 0 & 0 & 0 & RL8 \\
\hline
\end{tabular}

\section{B. Diseño y simulación del robot PA-10}

El diseño del robot PA-10 se realiza mediante el software CAD Solid Edge, permitiendo un diseño preliminar de los componentes cinemáticos del robot. Primero se diseñan las piezas del robot por separado en el entorno pieza; posteriormente, haciendo uso del entorno conjunto, se ensamblan las piezas que han sido creadas y se observa la interacción que existe entre estas, para posicionar el órgano terminal en una posición deseada (Fig. 5).

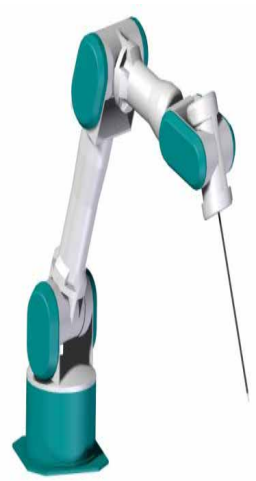

Fig. 5. Diseño del robot PA-10 en el software CAD.

Asimismo, este entorno permite configurar el tipo de material en que se construirá el robot y agregar los ejes de rotación al diseño realizado, facilitando el cálculo de los parámetros inerciales como la masa, el volumen y el centro de masa, con el fin de hallar los momentos de inercia necesarios para el modelo dinámico del robot (Tablas 2 y 3 ).

\section{TABLA 2}

VALORES DE LOS PARÁMETROS DINÁMICOS (PRIMER MOMENTO DE INERCIA)

\begin{tabular}{c|c|c|c|c|c|c}
\hline \multirow{2}{*}{ Articulaciones } & \multicolumn{2}{|c|}{$\begin{array}{c}\text { Momento de inercia en X } \\
\text { (Kg.m) }\end{array}$} & \multicolumn{2}{c|}{ Momento de inercia en Y } & \multicolumn{2}{c}{ Momento de inercia en Z } \\
& Mg.m) & \multicolumn{2}{c}{ (Kg.m) } \\
\hline Articulación 1 & MX1 & $-0,001304994$ & MY1 & 0,092329145 & MZ1 & 6,263547125 \\
\hline Articulación 2 & MX2 & $-0,001295329$ & MY2 & $-5,057899222$ & MZ2 & 1,107399869 \\
\hline Articulación 3 & MX3 & $-0,000439824$ & MY3 & 0,053981962 & MZ3 & 2,136038315 \\
\hline Articulación 4 & MX4 & $-0,000168414$ & MY4 & $-1,380103123$ & MZ4 & 0,506695466 \\
\hline Articulación 5 & MX5 & $-5,52544 \mathrm{E}-05$ & MY5 & 0,026990969 & MZ5 & 0,278344664 \\
\hline Articulación 6 & MX6 & $7,68857 \mathrm{E}-05$ & MY6 & $-0,017128284$ & MZ6 & 0,050611613 \\
\hline Articulación 7 & MX7 & $-1,42258 \mathrm{E}-07$ & MY7 & 0 & MZ7 & 0,000463004 \\
\hline
\end{tabular}

TABLA 3

VALORES DE LOS PARÁMETROS DINÁMICOS (SEGUNDO MOMENTO DE INERCIA)

\begin{tabular}{c|c|c|c|c|c|c}
\hline Articulaciones & \multicolumn{6}{|c}{ Momento de inercia en X, Y y Z $\left(\mathrm{Kg} \cdot \mathrm{m}^{2}\right)$} \\
\hline \multirow{2}{*}{ Articulación 1 } & Ixx & 3,958636584 & Iyy & 3,927444666 & Izz & 0,051621475 \\
\cline { 2 - 7 } & Ixy & $8,96572 \mathrm{E}-06$ & Ixz & $-0,000798989$ & Iyz & 0,039349258 \\
\hline \multirow{2}{*}{ Articulación 2 } & Ixx & 3,034902562 & Iyy & 0,14484614 & Izz & 2,904476538 \\
\cline { 2 - 7 } & Ixy & 0,000676965 & Ixz & $-0,000114148$ & Iyz & $-0,511540072$ \\
\hline \multirow{2}{*}{ Articulación 3 } & Ixx & 0,792444985 & Iyy & 0,773889573 & Izz & 0,0294865 \\
\cline { 2 - 7 } & Ixy & $1,24651 \mathrm{E}-05$ & Ixz & $-0,000182586$ & Iyz & 0,01473708 \\
\hline \multirow{2}{*}{ Articulación 4 } & Ixx & 0,482682499 & Iyy & 0,063293651 & Izz & 0,427046066 \\
\cline { 2 - 7 } & Ixy & $8,28407 \mathrm{E}-05$ & Ixz & $-2,04521 \mathrm{E}-06$ & Iyz & $-0,126755607$ \\
\hline \multirow{2}{*}{ Articulación 5 } & Ixx & 0,04282409 & Iyy & 0,033653767 & Izz & 0,013760459 \\
\cline { 2 - 7 } & Ixy & $1,3935 \mathrm{E}-05$ & Ixz & $-5,99829 \mathrm{E}-06$ & Iyz & 0,00264512 \\
\hline
\end{tabular}




\begin{tabular}{c|c|c|c|c|c|c}
\hline Articulaciones & \multicolumn{6}{|c}{ Momento de inercia en X, Y y Z $\left(\mathrm{Kg} \cdot \mathrm{m}^{2}\right)$} \\
\hline \multirow{2}{*}{ Articulación 6 } & Ixx & 0,007625747 & Iyy & 0,006506279 & Izz & 0,002435758 \\
\cline { 2 - 7 } & Ixy & $-2,01906 \mathrm{E}-06$ & Ixz & $7,97667 \mathrm{E}-06$ & Iyz & $-0,001455902$ \\
\hline \multirow{2}{*}{ Articulación 7 } & Ixx & 0,00011655 & Iyy & 0,000116549 & Izz & 0,00022816 \\
\cline { 2 - 7 } & Ixy & 0 & Ixz & $3,4305 \mathrm{E}-08$ & Iyz & $5,6 \mathrm{E}-12$ \\
\hline
\end{tabular}

Antes de pasar a la etapa de simulación, se hace necesario realizar un proceso de optimización para que el robot siga una trayectoria deseada, respetando la condición de cruce por el trocar [2]. Para ello se debe tener en cuenta la posición del trocar $\left(P_{t r}\right)$, que se encuentra entre la muñeca $\left(P_{m}\right)$ y el efector final $\left(P_{d}\right)$. Se requiere, entonces, que entre los tres puntos exista la condición de colinealidad, es decir, que estos puntos se encuentren alineados en cada instante de tiempo. Además de esto, hay que tener en cuenta la posición del codo $\left(P_{c}\right)$, la cual ayuda a posicionar la muñeca para cumplir con la condición de colinealidad [21].

Se utiliza, entonces, el algoritmo de Levenberg Mardquart, el cual es un algoritmo numérico iterativo de optimización que resuelve la función que proporciona las seis incógnitas articulares $\left(\theta_{1} \ldots \theta_{6}\right)$ [22]. Para ello se introducen las posiciones en $x, y$, $z$ de la consigna deseada al algoritmo de Levenberg Mardquart, del cual se obtienen las tres posiciones del codo $\left(P_{c}\right)$ y las tres de la muñeca $\left(P_{m}\right)$, las cuales garantizan el cruce por el trocar (obsérvese que la séptima articulación no tiene incidencia en este paso por el orificio abdominal). Mayores detalles sobre este proceso pueden verse en [21].
Con los modelos matemáticos obtenidos se realiza la simulación del robot PA-10 en el entorno MatlabSimulink; en estos se incluyen los valores numéricos de los parámetros inerciales obtenidos por medio de la herramienta CAD Solid Edge ${ }^{\circledR}$. Además, en la simulación se introducen los datos de las posiciones del codo y muñeca obtenidas en el proceso de optimización anterior, los cuales harán parte del modelo geométrico inverso del robot PA10. Se implementó un control por par calculado (computed torque control - CTC) [20] [23], el cual permite trabajar con una planta linealizada, obteniéndose los pares que irían a los motores del robot con miras a la adquisición futura de ellos para la posterior fabricación del robot. Se realizó un proceso de sintonización por ensayo y error para el seguimiento de diversas consignas, en dos y tres dimensiones. La Fig. 6 muestra el diagrama de control CTC implementado en simulación, y la Fig. 7, el error cartesiano del robot PA-10 al seguir una consigna circular efectuada en el plano XY, con 0,02 $\mathrm{m}$ de radio. El error máximo obtenido, de 1 milímetro, muestra un comportamiento aceptable del controlador.

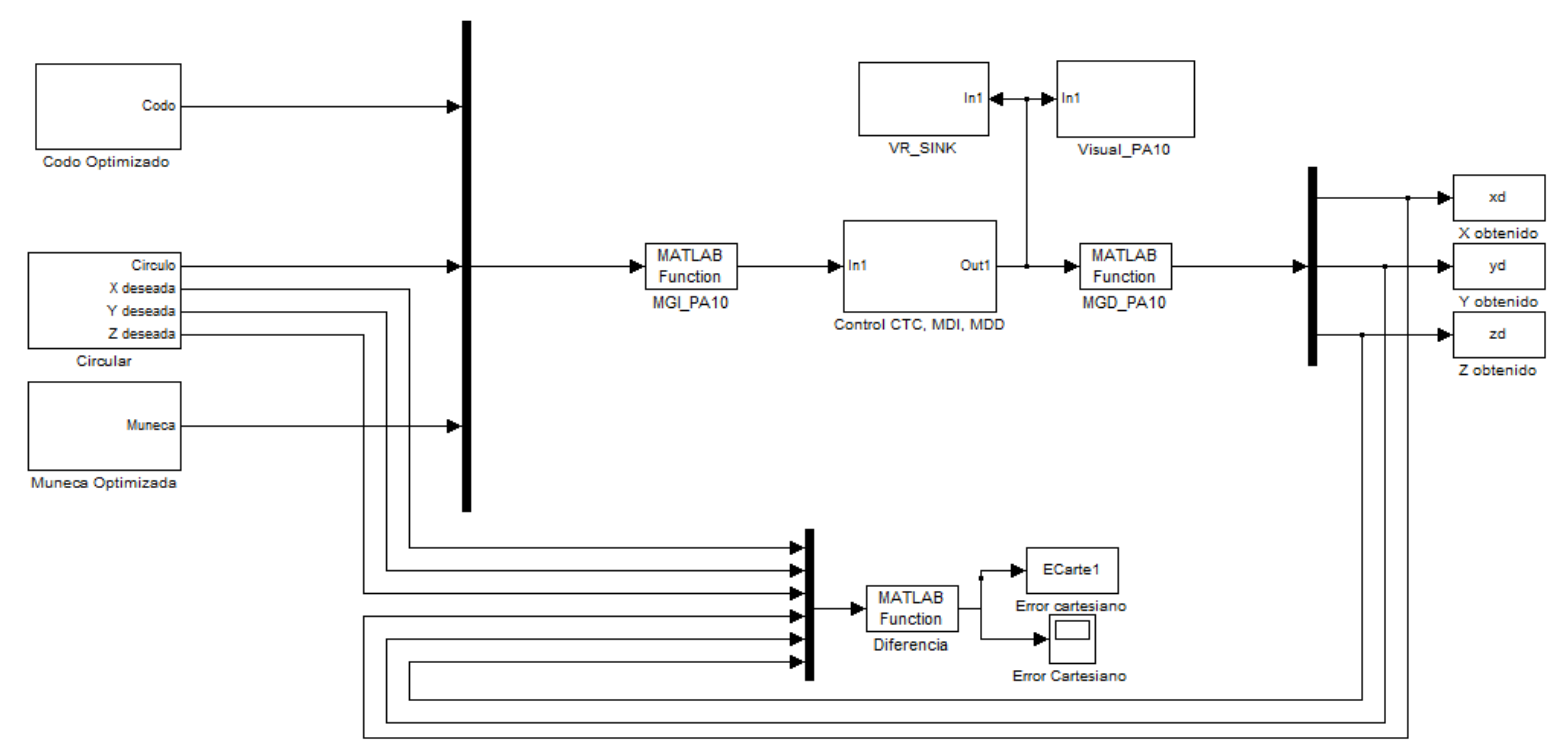

Fig. 6. Control por par calculado implementado sobre el robot PA-10. 


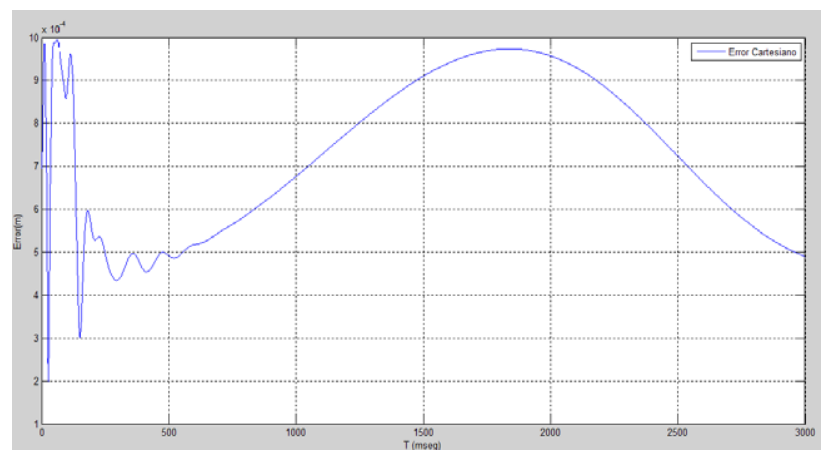

Fig. 7. Error cartesiano ante una consigna circular en el plano XY.

\section{SOFTWARE DE SIMULACIÓN VIRTUAL}

Se construyó la aplicación $S V R Q$ (Simulador Virtual de Robótica Quirúrgica), utilizando el motor gráfico VTK sobre Visual Studio 2010; este simulador contiene dos robots quirúrgicos (un Lapbot y un PA10), y un robot portaendoscopio (Hibou), movidos por joystick (Fig. 8).

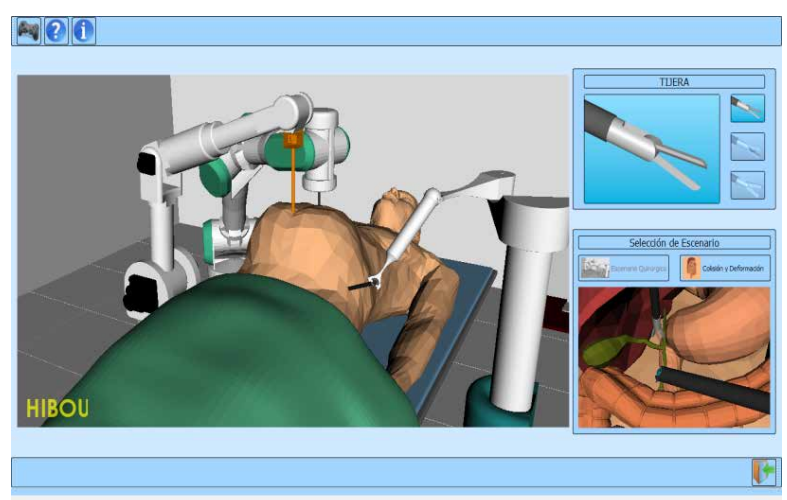

Fig. 8. Interfaz gráfica software SVRQ.

Las siguientes dos figuras (9 y 10) muestran los diagramas de casos de uso tanto del inicio de la aplicación, como de los dos escenarios con que ella trabaja (escenario quirúrgico y escenario de colisión y deformación).

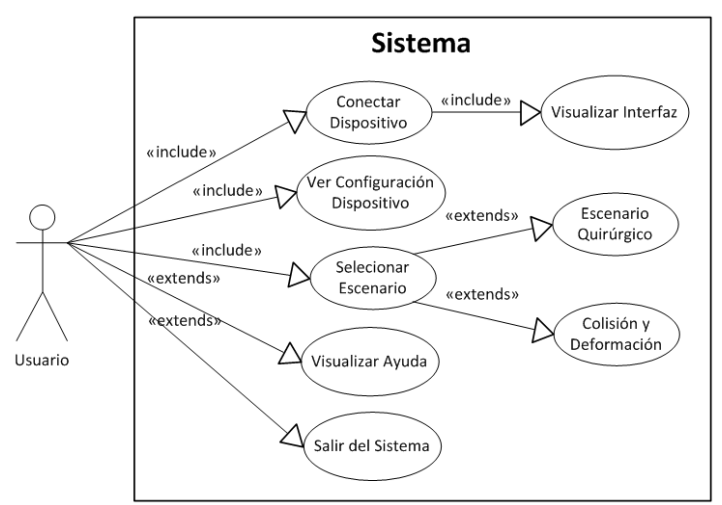

Fig. 9. Diagrama de casos de usos al inicializar la aplicación.

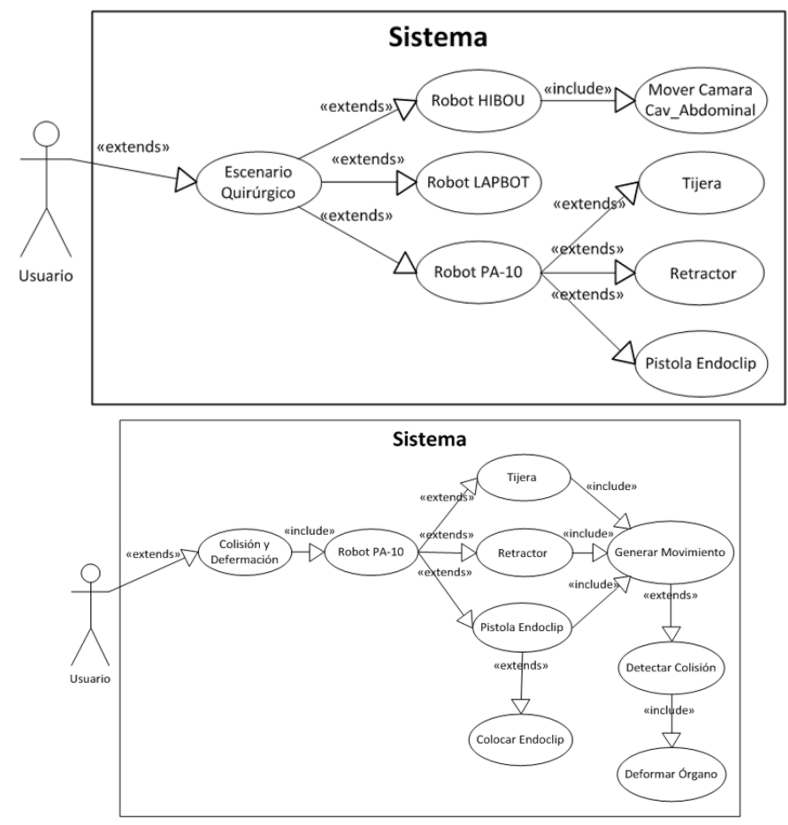

Fig. 10. Diagrama de casos de usos escenario quirúrgico y escenario de colisión y deformación. 


\section{A. Funcionamiento del software}

La aplicación $S V R Q$ tiene dos escenarios, como se observó anteriormente; en el primero ("Escenario Quirúrgico") existen tres ventanas: la primera presenta un ambiente donde se encuentra el paciente con los robots; en la segunda se observan tres herramientas quirúrgicas (tijera, retractor y pistola endoclip), las cuales son utilizadas por el robot tipo PA-10, y la tercera permite visualizar el interior de la cavidad abdominal del paciente, por medio de una cámara que es manipulada por el robot portaendoscopio Hibou. Al seleccionar el segundo escenario ("Colisión y Deformación"), la aplicación desplegará el ambiente mostrado en la Fig. 11.

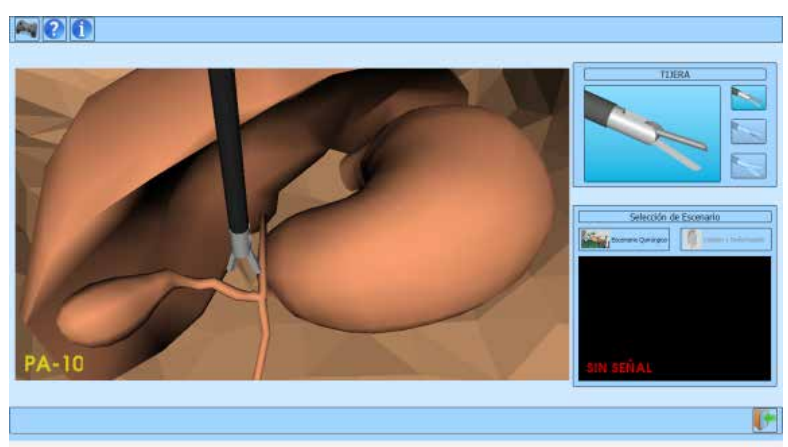

Fig. 11. Interfaz gráfica SVRQ (escenario Colisión y Deformación).

En el escenario "Colisión y Deformación", el usuario puede interactuar con los órganos de la cavidad abdominal, llevando el efector final del PA-10 a colisionar con los órganos y deformarlos si realiza una presión sostenida sobre él. Mayores detalles sobre los algoritmos de deformación utilizados pueden verse en [24].

\section{B. Colisión y deformación}

Para lograr que el efector final del robot tipo PA-10 interactúe con los órganos de la cavidad abdominal, se hace uso del algoritmo presentado en [24]. En este trabajo se explica cómo se verifica el contacto del órgano terminal del PA-10 con los órganos.

Para conocer la posición del órgano terminal del robot en la clase "Robot_PA_10", se define un método que retorne la "Matriz" instancia de la clase "vtkMatrix 4x4". Esta matriz almacena constantemente la posición del órgano terminal del robot, la cual es calculada por medio del modelo geométrico directo del robot.

Antes de almacenar los valores de posición del efector final en la matriz, se hace necesario efectuar un proceso de escalización, con el fin de acoplar los movimientos del órgano terminal a las posiciones cartesianas en las que se encuentra el órgano a deformar. Para ello hay que tener en cuenta la posición en que se ubican los objetos en el entorno Blender (herramienta donde se crearon los órganos), ya que estas posiciones afectan los órganos y al actor a la hora de colisionar. Por esta razón se realiza el siguiente procedimiento:

Primero se calcula el área de trabajo que recorre el órgano terminal, observando todos los movimientos que realiza la pinza, efectuados desde el dispositivo de mando; esto se puede observar en la Fig. 12, en cuyo espacio de trabajo se muestra la distancia recorrida en el eje $\mathrm{X}, \mathrm{Y}$ y Z, cuyo origen está situado en $\mathrm{X}=0.32 \mathrm{~m}$, $\mathrm{Y}=0.48 \mathrm{~m}, \mathrm{Z}=0 \mathrm{~m}$.

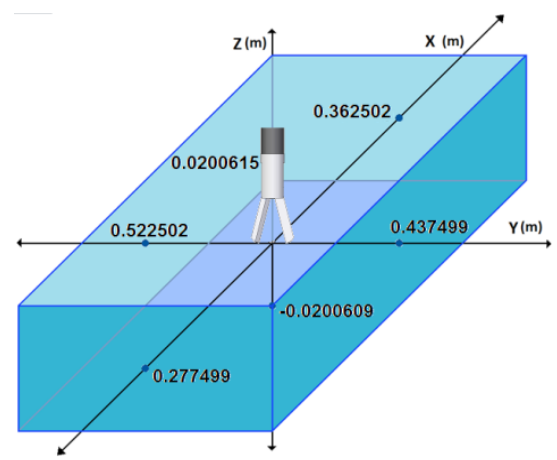

Fig. 12. Área de trabajo del órgano terminal.

Se calcula el área de trabajo que recorre el efector final con respecto a las posiciones cartesianas proporcionadas por Blender, llevando la pinza en el entorno de Blender a las posiciones que visualmente alcanza el efector final en el entorno SVRQ (Figura 13). El origen del plano cartesiano en Blender es $\mathrm{Xb}=$ $0 \mathrm{~m}, \mathrm{Yb}=0 \mathrm{~m}, \mathrm{y} \mathrm{Zb}=0 \mathrm{~m}$. 

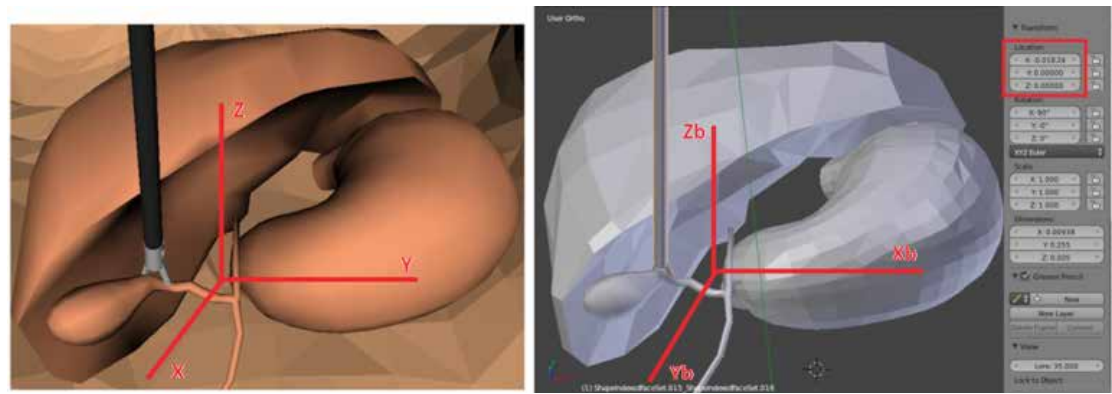

Fig. 13. Desplazamiento en SVRQ generando un desplazamiento en Blender.

En la Fig. 14 se comparan los planos cartesianos de los dos entornos; en esta se puede apreciar que un desplazamiento en el eje Y de SVRQ realiza un desplazamiento en el eje $\mathrm{Xb}$ de Blender, $\mathrm{y}$, de igual forma, un desplazamiento en el eje X de SVRQ genera un desplazamiento en el eje $\mathrm{Yb}$ de Blender. Cabe destacar que el eje Y de SVRQ se encuentra invertido con respecto al eje $\mathrm{Xb}$ de Blender, es decir, cuando Y alcance un Ymax en SVRQ, en Blender Xb alcanza un Xbmin. Para pasar del plano cartesiano de SVRQ al plano cartesiano de Blender se utiliza una matriz de transformación que permite realizar este procedimiento. Igualmente, se escalan los resultados para que los desplazamientos en Blender correspondan a los ejes adecuados en la aplicación SVRQ.
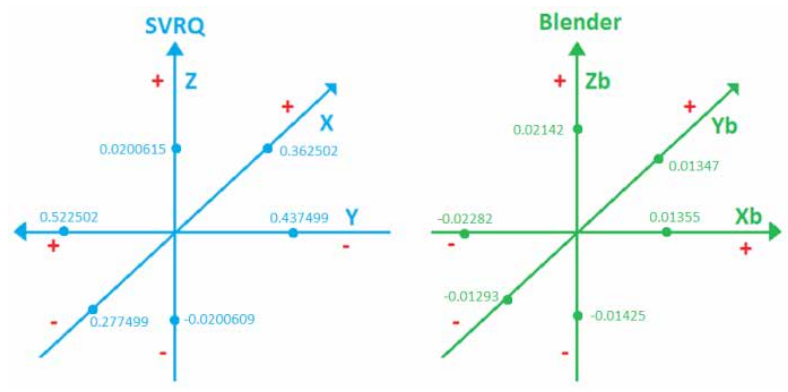

Fig. 14. Planos cartesianos en SVRQ y Blender.

Después de hallar las ecuaciones que transforman el desplazamiento del plano de SVRQ al plano de Blender es necesario tener en cuenta el plano de la "Matriz" instancia de la clase "vtkMatrix4x4" (Fig. $15)$.

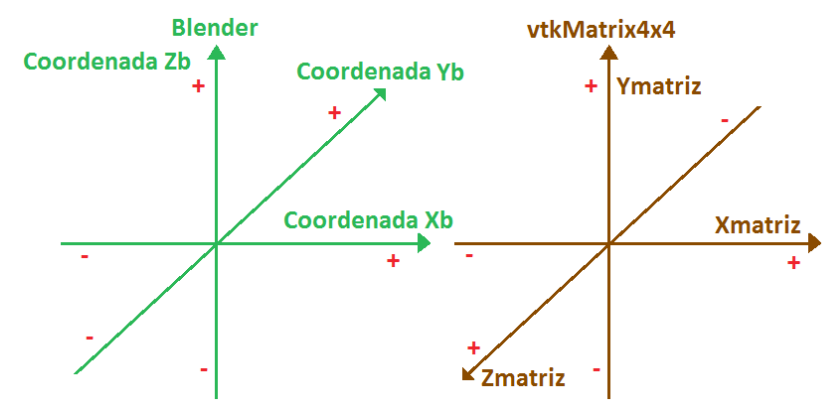

Fig. 15. Análisis de los planos cartesianos de Blender y de vtkMatrix $4 x 4$.

\section{Resultados}

La prueba de manipulación del robot PA-10 se llevó a cabo en el escenario quirúrgico de la aplicación SVRQ. Para manipular el robot se debe conectar el dispositivo de mando (joystick o gamepad), cuyos movimientos serán seguidos por el efector final del robot en el sentido que se le indique. A continuación (Fig. 16) se muestran los movimientos del órgano terminal del robot, en este caso en el eje $\mathrm{X}$, donde puede observarse que debido al paso por el trocar, al moverse el joystick hacia la derecha, el órgano terminal se mueve en esa dirección, pero el robot se moverá al lado contrario, y viceversa (efecto "fulcrum" o inversión de movimientos). 


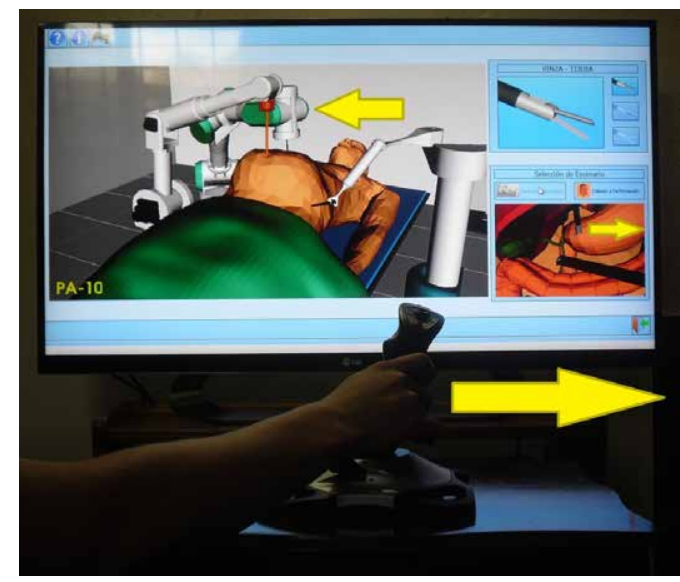

Fig. 16. Dispositivo joystick y órgano terminal moviéndose a la derecha.

La Fig. 17 presenta un ejemplo de deformación cuando el órgano terminal del robot PA-10, cargado con una "Tijera", toca el volumen del estómago. La Figura 18 muestra otra parte del procedimiento, cuando el robot PA-10 ha sido cargado con el instrumento "Pistola endoclip", que ha colocado los respectivos endoclips en el conducto cístico, con el fin de interrumpir la conexión entre el hígado y la vesícula, para posteriormente poder extraer esta última.

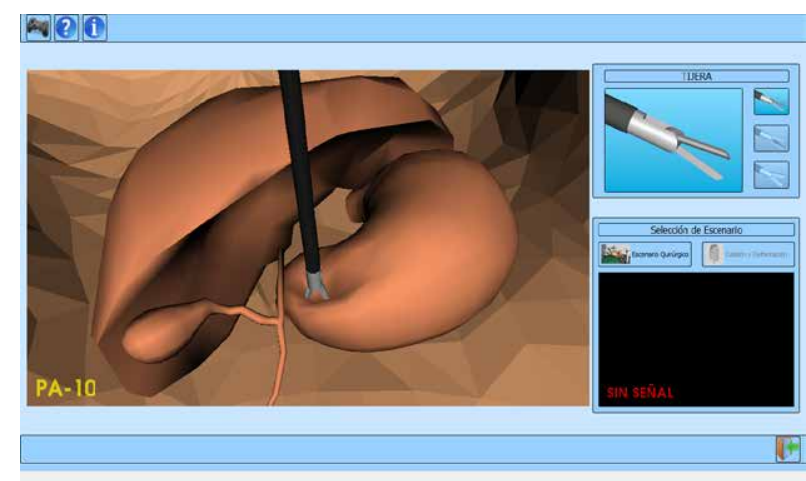

Fig. 17. Deformación del estómago con uno de los instrumentos del robot.

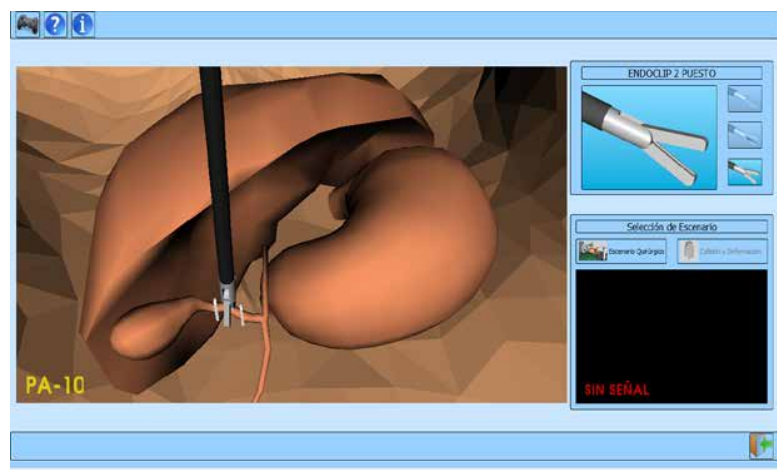

Fig. 18. Colocación de los endoclips en el conducto cístico.

Por último, la Fig. 19 muestra la propagación del movimiento sobre el estómago. Si se supone que el efector final del PA-10 impacta el triángulo en el centro de la propagación (color gris oscuro) y lo empieza a presionar hacia adentro, la propagación de movimiento primero se debe efectuar sobre los triángulos que están alrededor de él, es decir, los triángulos de color negro. Estos triángulos conforman el primer nivel de propagación. Si la presión se sigue efectuando sobre el triángulo gris oscuro, se van a ver también afectados los triángulos de color gris claro, que hacen parte del segundo nivel de propagación. El mismo comportamiento se sigue presentando hasta los triángulos del nivel $x$. Esta forma de propagación permite obtener el nivel de propagación de los vértices. Cabe destacar que este comportamiento se presenta para todos los polígonos triangulares que conforman el objeto.

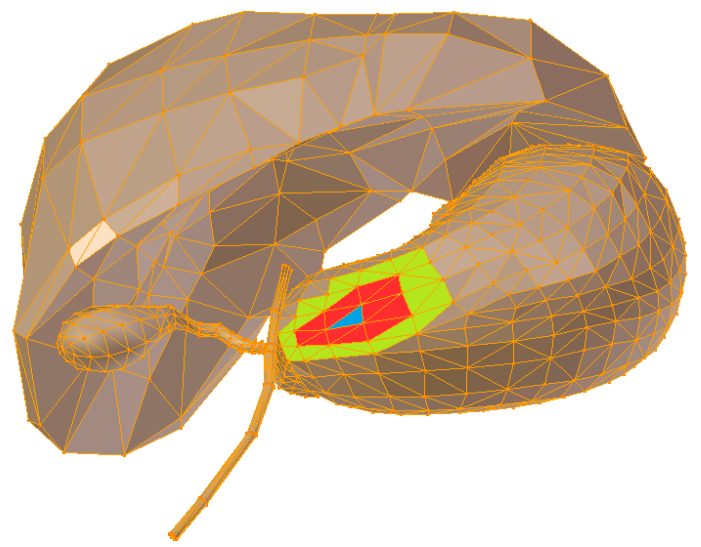

FIG. 19. Propagación del movimiento en un mallado triangular. 


\section{Conclusiones}

El ambiente virtual creado, llamado SVRQ, permite el entrenamiento del usuario para una colecistectomía laparoscópica (extracción de vesícula), pues el robot Hibou permite mover el endoscopio o cámara virtual con el fin de ubicar la zona de intervención, mientras que los dos robots quirúrgicos mueven los instrumentos necesarios en este tipo de cirugía (pinza, bisturí y cauterizador).

El software SVRQ recrea las cinco fases de la colecistectomía, proporcionando colisión y deformación del estómago, el hígado o la vesícula, al colisionar el instrumento portado por alguno de los dos robots quirúrgicos con dichos órganos.

Futuros trabajos implementarán otro tipo de cirugías laparoscópicas, además de aumentar el realismo en la simulación virtual.

\section{REFERENCIAS}

[1] R. Mishra, S. Wexner and R. Green, Textbook of Practical Laparoscopy Surgery. New Delhi: Jaypee Brothers Medical Publishers, 2013. DOI: http://dx.doi.org/10.5005/jp/books/11753.

[2] P. Escobar and T. Falcone, Atlas of SinglePort, Laparoscopic, and Robotic Surgery: A Practical Approach in Gynecology. New York: Springer New York, 2014. DOI: http://dx.doi. org/10.1007/978-1-4614-6840-0.

[3] G. H. Ballantyne and F. Molle, "The Da Vinci telerobotic surgical system: the virtual operative field and telepresence surgery", Surgical Clinics of North America, vol. 83 (6), pp. 1293-1304, Dec. 2003. DOI: http://dx.doi.org/10.1016/ S0039-6109(03)00164-6.

[4] R. Smith and M. Truong, Simulation in Robotic Surgery: a comparative review of simulators of the da Vinci surgical robot. Oviedo, FLA: Modelbenders Press, 2013.

[5] R. Polet and J. Donnez, "Using a laparoscope manipulator (Lapman) in laparoscopic gynecological surgery", Surgical Technology International, vol. 17, pp. 187-191, 2008.

[6] S. Kommu P. Rimington, C. Anderson and A. Rané, "Initial experience with the EndoAssist camera-holding robot in laparoscopic urological surgery", Journal of Robotic Surgery, vol. 1
(2), pp. 133-137, Jul. 2007. DOI: http://dx.doi. org/10.1007/s11701-007-0010-5.

[7] B. Kraft, C. Jäger, K. Kraft and R. Bittner, "The Aesop robot system in laparoscopic surgery: increased risk or advantage for surgeon and patient?", Surgical Endoscopy, vol. 18 (8), pp. 1216-1223, Aug. 2004. DOI: http://dx.doi. org/10.1007/s00464-003-9200-z.

[8] J. Justo, A. Pedroza, E. Prado et al., "Un nuevo simulador de laparoscopia", Medigraphic Artemisa, vol. 75 (1), pp. 19-23, Jan. - Feb. 2007.

[9] J. García, M. Arias and E. Valencia, "Diseño de prototipo de simulador para entrenamiento en cirugía laparoscópica", Revista Ingeniería Biomédica, vol. 5 (9), pp. 13-19, Jan. - Jun. 2011.

[10] S. Sanz, F. Sánchez, I. Díaz and J. Usón, "Validación preliminar del simulador físico Simulap ${ }^{\circledR}$ y de su sistema de evaluación para cirugía laparoscópica", Cirugía Española, vol. 90 (1), pp. 38-44, Jan. 2012. DOI: http://dx.doi. org/10.1016/j.ciresp.2011.07.013.

[11] T. Matsuda, Y. Ono, S. Baba et al., "Positive correlation between motion analysis data on the Lapmentor virtual reality laparoscopic surgical simulator and the results from video tape assessment of real laparoscopic surgeries", The Journal of Urology, vol. 179 (4), p. 661, Apr. 2008. DOI: http://dx.doi.org/10.1016/S00225347(08)61930-8.

[12] A. Rolls, C. Riga, C. Bicknell et al., "A pilot study of video-motion analysis in endovascular surgery: Development of real-time discriminatory skill metrics", European Journal of Vascular and Endovascular Surgery, vol. 45 (5), pp. 509-515, May. 2013. DOI: http://dx.doi. org/10.1016/j.ejvs.2013.02.004.

[13] S. Patel, C. Radia, S. Bains and R. Varma, "WII Trauma Centre VS. LapSim: Are modern game consoles comparable to simulators for the development of laparoscopic skills?", International Journal of Surgery, vol. 11 (8), p. 598, Oct. 2013. Sapna Patel, Chandni Radia, Sonia Bains, Rajesh Varma. DOI: http://dx.doi. org/10.1016/j.ijsu.2013.06.062.

[14] W. Brinkman, J. Luursema, B. Kengen et al., "Da Vinci skills simulator for assessing learning curve and criterion-based training of robotic basic skills", Urology, vol. 81 (3), pp. 562-566, Mar. 2013. DOI: http://dx.doi.org/10.1016/j. urology.2012.10.020. 
[15] D. Guzmán and A. Vivas, "Software para la práctica de la robótica quirúrgica", Ingeniería y Universidad, vol. 19 (1), pp. 7-25, Jan. Jun. 2015. DOI: http://dx.doi.org/10.11144/ Javeriana.iyu19-1.sprq.

[16] S. Salinas and A. Vivas, "Modelado, simulación y control del robot para cirugía laparoscópica Lapbot", Revista Chilena de Ingeniería, vol. 17 (3), pp. 317-328, Dec. 2009. DOI: http://dx.doi. org/10.4067/s0718-33052009000300005.

[17] V. Torres, C. Méndez, A. Vivas and S. Salinas, "Diseño y simulación en 3D de un robot porta endoscopio para operaciones de laparoscopia", en $V$ Seminario Internacional de Ingeniería Electrónica, Bucaramanga, Colombia, Sep. 2011.

[18] C. W. Kennedy and J. P. Desai, "Modeling and control of the Mitsubishi PA10 robot arm harmonic drive system", IEEE ASME Transactions on Mechatronics, vol. 10 (3), pp. 263-274, Jun. 2005. DOI: http://dx.doi. org/10.1109/TMECH.2005.848290.

[19] N. Bompos, P. Artemiadis, A. Oikonomopoulos and K. Kyriakopoulos, "Modeling, full identification and control of the mitsubishi
PA-10 robot arm", IEEE/ASME International Conference on Advanced Intelligent Mechatronics, Zurich (Switzerland), pp. 1-6, Sep. 2007. DOI: http://dx.doi.org/10.1109/ aim.2007.4412421.

[20] E. Dombre and W. Khalil, Modeling, Performance Analysis and Control of Robot Manipulators. London: Wiley, 2010.

[21] B. Garcés, O. Mora and A. Vivas, "Estudio del uso de robots industriales como asistentes en operaciones de laparoscopia", Revista Facultad de Ingeniería Universidad de Antioquia, $\mathrm{n}^{\circ}$ 47, pp. 91-102, Mar. 2009.

[22] K. Levenberg, "A method for the solution of certain nonlinear problems in least squares", Quarterly of Applied Mathematics, vol. 2, pp. 164-168, 1944.

[23] A. Vivas, Diseño y Control de Robots Industriales: Teoría y Práctica. Buenos Aires: Elaleph, 2010.

[24] R. Gómez and F. Montero, "Visualización y deformación de objetos virtuales 3d", Tesis de grado, Ingeniería en Automática Industrial, Universidad del Cauca, Colombia, 2012. 